\title{
Role of Major Glucosinolates in the Defense of Kale Against Sclerotinia sclerotiorum and Xanthomonas campestris pv. campestris
}

\author{
Pari Madloo, ${ }^{1,2}$ Margarita Lema, ${ }^{2}$ Marta Francisco, ${ }^{1}$ and Pilar Soengas ${ }^{1, \dagger}$ \\ ${ }^{1}$ Group of Genetics, Breeding and Biochemistry of Brassicas, Misión Biológica de Galicia (MBG-CSIC), Spanish Council for Scientific \\ Research, Pontevedra, Spain \\ 2 Department of Functional Biology, Faculty of Biology, University of Santiago de Compostela, Spain \\ Accepted for publication 27 March 2019.
}

\begin{abstract}
Glucosinolates (GSLs) are secondary metabolites present in Brassicaceae species implicated in their defense against plant pathogens. When a pathogen causes tissue damage, the enzyme myrosinase hydrolyzes GSLs into diverse products that exhibit antimicrobial activity against a wide range of bacteria and fungi in vitro. It was demonstrated that modulation of GSL content in vivo affects plant resistance to infection by pathogens in Arabidopsis. However, the roles of specific metabolites and how they interact with pathogens are poorly understood in Brassica crops. We previously developed a set of populations of Brassica oleracea var. acephala L. (kale) differing in content of three GSLs: the aliphatics sinigrin (2-propenyl [SIN]) and glucoiberin (3-methylsulphinylpropyl [GIB]) and the indolic glucobrassicin (3-indolylmethyl [GBS]). These populations can be used to study the effects of major GSLs in kale, with the advantage that genotypes within each selection have the same genetic background. This research
\end{abstract}

ABSTRACT aimed to explore the role of SIN, GIB, and GBS in the defense of kale against the necrotrophic fungus Sclerotinia sclerotiorum and the bacterium Xanthomonas campestris pv. campestris. Results showed that increasing the amount of a particular GSL did not always result in disease resistance. The effects of GSLs were apparently dependent on the pathogen and the type of GSL. Thus, the aliphatic SIN was inhibitory to infection by S. sclerotiorum and the indolic GBS was inhibitory to infection by $X$. campestris pv. campestris. Other factors, including the quantity and proportion of other metabolites modified during the pathogen infection process, could also modulate the degree of inhibition to the pathogen.

Keywords: biochemistry and cell biology, black rot, Brassica oleracea, disease control and pest management, genetics and resistance, plant resistance, secondary metabolites, white mold
Plants defend themselves against pathogens by synthetizing and accumulating chemically diverse metabolites with antibiotic properties (Pastorczyk and Bednarek 2016). Constitutive synthesis and accumulation of defensive metabolites (phytoanticipins) is resource demanding; therefore, de novo synthesis can be induced after plant tissue is exposed to pathogen infection (phytoalexins) (Pastorczyk and Bednarek 2016). Brassicaceae species accumulate up to $1 \%$ of dry weight as the sulfur-containing metabolites glucosinolates (GSLs) (Calmes et al. 2015). GSLs can be classified into three chemical classes, depending on the side chain of their precursor amino acid. Methionine, alanine, valine, leucine, and isoleucine are precursors of aliphatic GSLs. Phenylalanine and tyrosine render aromatic GSLs and tryptophan is the precursor of indolic GSLs (Zukalova and Vasak 2002). They are constitutively synthetized and stored in plant cells, functioning mainly as phytoanticipins, although they can be induced in response to a variety of stimuli, as well as following exposure to insect damage and pathogen infection (Brader et al. 2006; Buxdorf et al. 2013). De novo synthesis of indolic GSLs in Arabidopsis can be triggered by plant pathogens, at the same time inducing synthesis of the phytoalexin camalexin through the upregulation of the genes CYP79B2 and CYP79B3, which are shared by several indolic pathways (Brader et al. 2006; Frerigmann et al. 2016; Stahl et al. 2016).

${ }^{\dagger}$ Corresponding author: P. Soengas; psoengas@mbg.csic.es

Funding: This research was supported by the Spanish Ministry of Economy and Competitiveness through the project AGL2015-66256-C2-1. P. Madloo acknowledges a Ph.D. student fellowship from Erasmus Mundus Action 2 Program.

The author(s) declare no conflict of interest.

(C) 2019 The American Phytopathological Society
Myrosinase enzymes hydrolyze GSLs into several chemically diverse active products. Myrosinases and GSLs are stored in different plant compartments and, after tissue damage caused by injuries, pests, or pathogens (Calmes et al. 2015), these constituents mix, and hydrolysis of GSLs occurs. Hydrolytic products exhibit antimicrobial activities against a wide range of bacterial and fungal plant pathogens in vitro (Aires et al. 2009; Calmes et al. 2015; Dufour et al. 2015; Li et al. 2013; Sotelo et al. 2014a; Stotz et al. 2011). Antimicrobial effects depend on the dose, pathogen, and specific race or isolate (Sotelo et al. 2014a). In addition, the chemical structure of the breakdown products determine their activity in vitro, with isothiocyanates (ITCs) being the most toxic, even at low concentrations (Pastorczyk and Bednarek 2016). Toxicity of ITCs is also related to the length of their side chain ( $\mathrm{Li}$ et al. 2013).

In planta activation of GSLs is dependent on tissue breakdown; however, this does not happen in the interaction with biotrophic pathogens (Calmes et al. 2015). Production and liberation of active indole compounds to the apoplast can also be mediated by the action of the genes pen 2 and pen3, as described with the biotroph fungus Blumeria graminis f. sp. hordei (Johansson et al. 2014). To demonstrate the role of GSLs in the immunity of Brassicaceae plants to various pathogens, knockout mutants of genes implicated in the synthesis and hydrolysis of GSLs in Arabidopsis have been tested against various bacteria and fungi. The double mutant CYP79B2/B3, which does not accumulate indolic GSLs or camalexin, and the double mutant MYB28/29, which does not accumulate aliphatic GSLs, modified the sensitivity of plants against isolates of the fungi Alternaria brassicicola (Schwein.) Wiltshire, Botrytis cinerea Pers. (Buxdorf et al. 2013), and Sclerotinia sclerotiorum (Lib.) de Bary (Stotz et al. 2011). However, transgenic Arabidopsis plants accumulating specific GSLs can increase their resistance to pathogens. Expression of the CYP79D2 gene from cassava in Arabidopsis causes accumulation of aliphatic 
isopropyl and methylpropyl GSLs that enhance resistance to the bacteria Erwinia carotovora (van Hall) Dye. Arabidopsis expressing the sorghum CYP79A1 or overexpressing the endogenous CYP79A2 accumulate p-hydroxybenzyl or benzyl GSLs, respectively, increasing resistance to Pseudomonas syringae van Hall (Brader et al. 2006). The contribution of GSLs to the defense of Arabidopsis is dependent on the pathogen species and even on the isolate under consideration (Brader et al. 2006; Pastorczyk and Bednarek 2016). Specialist pathogens can adapt themselves by detoxifying ITCs or suppressing the induction of GSLs biosynthesis (Pastorczyk and Bednarek 2016). Variability in the response among pathogens may also be due to different life styles (i.e., biotrophic versus necrotrophic) or differential host ranges (Buxdorf et al. 2013).

Brassica crops are affected by several diseases that can reduce the quantity and quality of yield, resulting in economic losses worldwide (Kayum et al. 2016). Research efforts are dedicated to identifying resistant materials and discovering the mechanisms underlying that resistance. However, knowledge of the role of specific metabolites in defense of Brassica crops against pathogens is not well characterized. The content of GSLs in several varieties of Brassica oleracea and B. napus has been studied in relation to resistance to Xanthomonas campestris pv. campestris (Pammel) Dowson, S. sclerotiorum, and B. cinerea (Aires et al. 2011; Giamoustaris and Mithen 1997). However, it should be noted that the host genotypes had different GSL profiles and concentrations, as well as different genetic backgrounds. Recent work found that lines of B. napus, which overexpressed UGT74B, an indolic GSL pathway gene, enhanced not only the production of total indolic GSLs but also the resistance to $S$. sclerotiorum and $B$. cinerea (Zhang et al. 2015).

Although these efforts show that modulating the content of GSLs may affect Brassica plant resistance to pathogens, the contribution of specific metabolites is poorly understood. Previously, we used mass selection to develop a set of B. oleracea var. acephala L. (kale) genotypes differing in the content of three GSLs: the aliphatics sinigrin (2-propenyl [SIN]) and glucoiberin (3methylsulphinylpropyl [GIB]) and the indolic glucobrassicin (3-indolylmethyl [GBS]). Sotelo et al. (2016) evaluated these genotypes and concluded that different alleles at the GSL-ALK locus were responsible for variation in the content of SIN and GIB, whereas the CYP81F2 gene may be responsible for the variation in concentration of GBS. This material can be used to study the effects of major GSLs in kale, with the advantage that the genotypes all have the same genetic background (Sotelo et al. 2016). The aim of this research was to assess the role of SIN, GIB, and GBS in the defense of kale plants against the necrotrophic pathogens $S$. sclerotiorum and X. campestris pv. campestris.

\section{MATERIALS AND METHODS}

Plant and pathogen culture. Three independent divergent selection programs were performed using mass selection at the Misión Biológica de Galicia, Spanish Council for Scientific Research (MBG-CSIC), Spain, so as to obtain genotypes of B. oleracea differing in content of three major GSLs (namely, SIN, GIB, and GBS) (Sotelo et al. 2016). The three divergent selection programs were all based on the landrace MBG-BRS0062. This is a heterogeneous kale variety, cultivated by farmers since ancient times in the northwest of the Iberian Peninsula (Padilla et al. 2007; Tortosa et al. 2017). The race is maintained at the germplasm bank at the MBG-CSIC. MBG-BRS0062 was chosen because it had broad genetic variability in GSL content, a desirable characteristic on which to perform selection (Sotelo et al. 2016). For each one of the divergent selections (SIN, GIB, and SIN), three consecutive cycles of selection were performed to obtain genotypes with high (H) or low (L) GSL content. Six genotypes were obtained: HSIN, LSIN, HGIB, LGIB, HGBS, and LGBS, which were used to test the effect of SIN, GIB, and GBS on infection of kale by two pathogens (X. campestris pv. campestris and S. sclerotiorum) of B. oleracea.

The isolate of $X$. campestris pv. campestris belongs to race 1 (strain HRI3811, synonymous with PHW1205 collected from B. oleracea in the United States) (J. Vicente personal communication) and was provided by Joana Vicente (Warwick HRI, Wellesbourne, U.K.). Fresh bacterial colonies of X. campestris pv. campestris were subcultured on Petri dishes containing potato dextrose agar (PDA) and incubated at $32^{\circ} \mathrm{C}$ for $24 \mathrm{~h}$ in the dark. For inoculum preparation, a loop of bacteria was transferred to nutrient broth and shaken overnight at $150 \mathrm{rpm}$ and $30^{\circ} \mathrm{C}$ in the dark. The culture was diluted in sterile tap water to a concentration of $5 \times 10^{8}$ $\mathrm{CFU} \cdot \mathrm{ml}^{-1}$, which corresponds to an absorbance of 0.51 at a wavelength of $600 \mathrm{~nm}$, measured using a spectrophotometer (Spectra MR; Dynex Technologies, Chantilly, VA, U.S.A.). The S. sclerotiorum isolate MBG-Ss2 was provided by MBG-CSIC. The original isolate was collected in January 2008 from a naturally infected plant of B. napus in an experimental field at MBG $\left(42^{\circ} 24^{\prime} 23^{\prime \prime} \mathrm{N}, 8^{\circ} 38^{\prime} 31^{\prime \prime} \mathrm{W}\right)$.

Fresh colonies of $S$. sclerotiorum were obtained through routine transfer of mycelium-agar plugs from the margin of a colony of $S$. sclerotiorum growing on PDA medium. The cultures were incubated with a 14-h photoperiod and day and night mean temperatures of 24 and $18^{\circ} \mathrm{C}$, respectively. Agar plugs $(4 \mathrm{~mm}$ in diameter) containing the advancing edge of fungal mycelia were used as the inoculum source.

Plant-inoculation and disease severity measurements. The reactions of six kale genotypes (LSIN, HSIN, LGBS, HGBS, LGIB, and HGIB) to infection by $X$. campestris pv. campestris and $S$. sclerotiorum were tested in two independent experiments in a greenhouse with a 14-h photoperiod; day and night mean temperatures of 24 and $18^{\circ} \mathrm{C}$, respectively; and $70 \%$ relative humidity. Plants were grown in pots containing 2.5 liters of peat (Gramoflor GmbH \& Co. KG Produktion, Vechta, Germany). In both experiments, 30 plants were inoculated per genotype in a completely randomized design. Variability among the 30 individuals, which were genetically different, was considered as the error in both experiments. Six weeks after sowing, the second-youngest leaf of each plant (counting from the apex) was inoculated. This was considered the first trial of the experiments (T1).

For $X$. campestris pv. campestris, inoculum was injected into three different points of each leaf, puncturing the main veins using mouse-tooth forceps wrapped in cotton soaked in the bacterial suspension. After inoculation, plants were placed in the greenhouse with a day/night mean temperature of $24 / 28^{\circ} \mathrm{C}$, a $14 \mathrm{~h}$ photoperiod and a relative humidity of 90 to $100 \%$. At 21 days postinoculation, the inoculated leaves were collected and photographed. The area of each chlorotic lesion was measured using ImageJ software version $1.51 \mathrm{n}$ (LOCI; University of Wisconsin, Madison, WI, U.S.A.). Total lesion area (in square centimeters) was calculated by summing the area of all the individual chlorotic lesions on a leaf. For the S. sclerotiorum experiment, leaves were inoculated by placing one agar plug with the fungal inoculum on the upper side of the leaf. On the fourth day postinoculation, inoculated leaves were collected and photographed, and the area of necrotic lesion per leaf was obtained with ImageJ software, as described above. Five replicate plants per genotype were used as controls (not inoculated).

Subsequent to collecting the inoculated leaves and control leaves in both the $X$. campestris pv. campestris and $S$. sclerotiorum experiments in $\mathrm{T} 1$, the second-youngest leaf of each plant counting from the apex was inoculated as described above for T1. All experiment procedures were the same as described above for T1. This was considered the second trial (repeat) of the experiments (T2).

GSL identification and quantification. After photographing inoculated leaves at T1 and T2, they were immediately frozen in solid dry ice, transferred to the laboratory, and stored at $-80^{\circ} \mathrm{C}$. GSL extraction followed the methodology of Kliebenstein et al. (2001), with minor modifications to perform multiple extractions in 
96 plates. Identification and quantification of compounds was performed using ultrahigh performance liquid chromatography according to Sotelo et al. (2014b). GSL concentration was measured in micromoles per gram dry weight.

Data analyses. The variables lesion area and GSL concentration were subjected to analysis of variance using a generalized linear model. Analysis of variance were performed independently by experiment (plants inoculated with S. sclerotiorum or $X$. campestris pv. campestris) and by trial (T1 and $\mathrm{T} 2$ ) within each experiment. GSL genotypes (high or low) and individual plants were considered sources of variation. Genotypes were considered fixed effects, whereas replications (plants within each GSL selection) were considered random factors. For each experiment and trial, three separate analyses of variance were performed. First, genotypes for each divergent selection (SIN, GIB, and GBS) and controls were analyzed. Second, only genotypes for each divergent selection were included. Third, an analysis comparing genotypes among divergent selections was performed. All analyses were completed using R Core Team (2018) and the Rcmdr package (Fox and Bouchet-Valata 2018). Means comparisons were performed for all traits using Fisher's protected least significant difference at $\alpha=0.05$.

\section{RESULTS}

Disease severity and its relationship to the target and other GSLs within each selection. Disease severity in the three divergent selections was measured as the area of the lesion caused by $S$. sclerotiorum or $X$. campestris pv. campestris in two independent experiments. GSL concentration was measured in the same leaves as disease severity. In addition to the three major GSLs under selection, a further three GSLs were detected across the six genotypes in the control plants in S. sclerotiorum experiment: the indolics hidroxyglucobrassicin (4-hydroxy-3-indolylmethyl [OHGBS]), neoglucobrassicin (1-methoxy-3-indolylmethyl [NEOGBS]), and methoxyglucobrassicin (4-methoxy-3-indolylmethyl [MEGBS]) (Fig. 1). The same GSLs were identified in the six genotypes inoculated with $S$. sclerotiorum or $X$. campestris pv. campestris in both T1 and T2 (Figs. 2, 3, and 4). We present the results in three sections related to the divergent selections to describe the relationship between disease severity and content of each GSL: SIN, GBS, GIB, and the additional GSLs.

To determine whether there was induction of GSLs after inoculation with S. sclerotiorum, the concentration of GSLs of inoculated plants was compared with that of controls in the three divergent selections. No difference was found in the profile of GSLs; however, significant differences among GSL concentrations were detected (SIN selection T1: SIN: $P<0.001$; MEGBS: $P=$ 0.001; OHGBS: $P=0.02$; SIN selection T2: SIN: $P<0.001$; MEGBS: $P=0.03$; OHGBS: $P=0.02$; GIB selection T1: GIB: $P<0.001$; MEGBS: $P<0.001$; NEOGBS: $P=0.005$; OHGBS: $P<$ 0.001; GIB selection T2: SIN: $P=0.02$; GIB: $<0.001$; MEGBS: $P<$ 0.001; OHGBS: $P<0.001$; GBS selection T1: GBS: $P=0.009$; MEGBS: $P=0.001$; GBS selection T2: SIN: $P<0.001$; GBS: $P=$ 0.002; NEOGBS: $P=0.02$; NEOGBS: $P<0.001$; OHGBS: $P=$ 0.04) (Table 1). Generally speaking, the indolics MEGBS and NEOGBS were induced in inoculated plants compared with controls (Fig. 1A to C), although this is more apparent in the GIB-selected genotype compared with the SIN or GBS genotypes. The aliphatics SIN and GIB were actually repressed in T2 in the inoculated plants of the GIB selection (Fig. 1B).

SIN-selected plants. Subsequent to inoculation with S. sclerotiorum, there were significant differences between HSIN and LSIN for lesion area in both trials $(P=0.05$ and $P<0.001$ in T1 and T2, respectively) (Table 2). Plants with HSIN had significantly smaller lesion areas in both $\mathrm{T} 1$ and $\mathrm{T} 2$ compared with plants with LSIN (Fig. 2A). In contrast, those HSIN plants inoculated with $X$. campestris pv. campestris had larger lesions compared with the
LSIN plants in T1 but a similar response in T2. SIN concentration was significantly higher in HSIN plants compared with LSIN plants in T2 but the difference was not significant in T1 (Fig. 2B and $\mathrm{C}$ ).

The concentration of the indolics MEGBS and NEOGBS was significantly higher in S. sclerotiorum-inoculated HSIN plants compared with $S$. sclerotiorum-inoculated LSIN plants in T1 and T2, respectively. OHGBS was significantly higher in LSIN-selected plants compared with HSIN-selected plants in T2 (Fig. 2B). When SIN-selected plants were inoculated with $X$. campestris $\mathrm{pv}$. campestris, OHGBS concentration was higher in HSIN plants, whereas MEGBS and NEOGBS were higher in LSIN plants in T1 (Fig. 2C).

GIB-selected plants. When GIB-selected plants were inoculated with $S$. sclerotiorum, there were significant differences $(P<0.001)$ between genotypes for lesion area in $\mathrm{T} 1$ in the analysis of variance (Table 2). HGIB plants had a smaller lesion area in T1 when compared with lesion size on the LGIB plants (Fig. 3A). GIB content was significantly higher in the HGIB plants compared with the LGIB plants in both T1 and T2 (Fig. 3B). Furthermore, SIN content was higher in LGIB plants in T1 and OHGBS was higher in HGIB plants in both T1 and T2. There were no significant differences between genotypes for lesion area when inoculated with X. campestris pv. campestris (Table 2). However, the content of GIB was significantly higher in HGIB plants compared with that in LGIB plants in both T1 and T2. The concentration of OHGBS was significantly higher in HGIB plants compared with LGIB plants in T1, whereas SIN was lower in HGIB plants when compared with that in LGIB plants in T2 (Fig. 3C).

GBS-selected plants. No significant differences among genotypes were found when plants of the GBS selection were inoculated with $S$. sclerotiorum (Table 2). When genotypes were inoculated with $X$. campestris pv. campestris, the HGBS plants exhibited a resistant response, with noticeably smaller lesion areas (Fig. 4A) compared with the LGBS plants in both T1 and T2. HGBS plants had a significantly higher concentration of GBS when compared with LGBS plants in both experiments in both T1 and T2. However, the total concentration of GBS and the difference in GBS concentration between the HGBS plants and the LGBS plants was higher when plants were inoculated with $S$. sclerotiorum than when inoculated with $X$. campestris pv. campestris (Fig. 4B and C). In GBS-selected plants, the HGBS plants that were inoculated with $S$. sclerotiorum had significantly higher concentrations of GIB in T1 when compared with the LGBS plants. The HGBS plants had a higher concentration of SIN and NEOGBS in T2, whereas MEGBS was higher in the LGBS plants. In both T1 and T2, the concentration of NEOGBS was significantly higher in HGBS plants inoculated with $X$. campestris pv. campestris compared with the LGBS plants inoculated with $X$. campestris pv. campestris.

Disease severity and its relationship to SIN, GBS, GIB, and the additional GSLs. There were significant differences for lesion area in the analysis of variance among the three selections when plants were inoculated with $S$. sclerotiorum $(P<0.001$ in T1 and T2) or $X$. campestris pv. campestris $(P=0.006$ in $\mathrm{T} 1$ and $P=$ 0.04 in T2) (Table 3). In plants inoculated with $S$. sclerotiorum, the LGIB plants had significantly larger lesions compared with the other genotypes in T1 (Fig. 5A). The HSIN plants exhibited a significantly smaller lesion area compared with the other genotypes, with the exception of lesions on the LGBS plants in T2. The HSIN plants had significantly more SIN compared with the other genotypes in T2. Furthermore, the NEOGBS content of HSIN plants was numerically highest, although it was not significantly different from that in the other genotypes. However, the concentration of GSLs cannot explain the relatively small lesion size observed in LGBS plants, which have low SIN and NEOGBS content (Fig. 5A). 
In plants inoculated with $X$. campestris pv. campestris, the HGBS plants had smaller lesions compared with the other genotypes, although the lesion size did not differ significantly from those on the LSIN, HGIB, and LGIB plants in T1 or from the lesions on the HSIN,
LSIN, and HGIB plants in T2 (Fig. 5B). Thus, the resistance exhibited by HGBS plants to $X$. campestris pv. campestris compared with the other genotypes cannot be explained by GBS content, because the HGBS plants had a low GBS content in both T1 and T2.

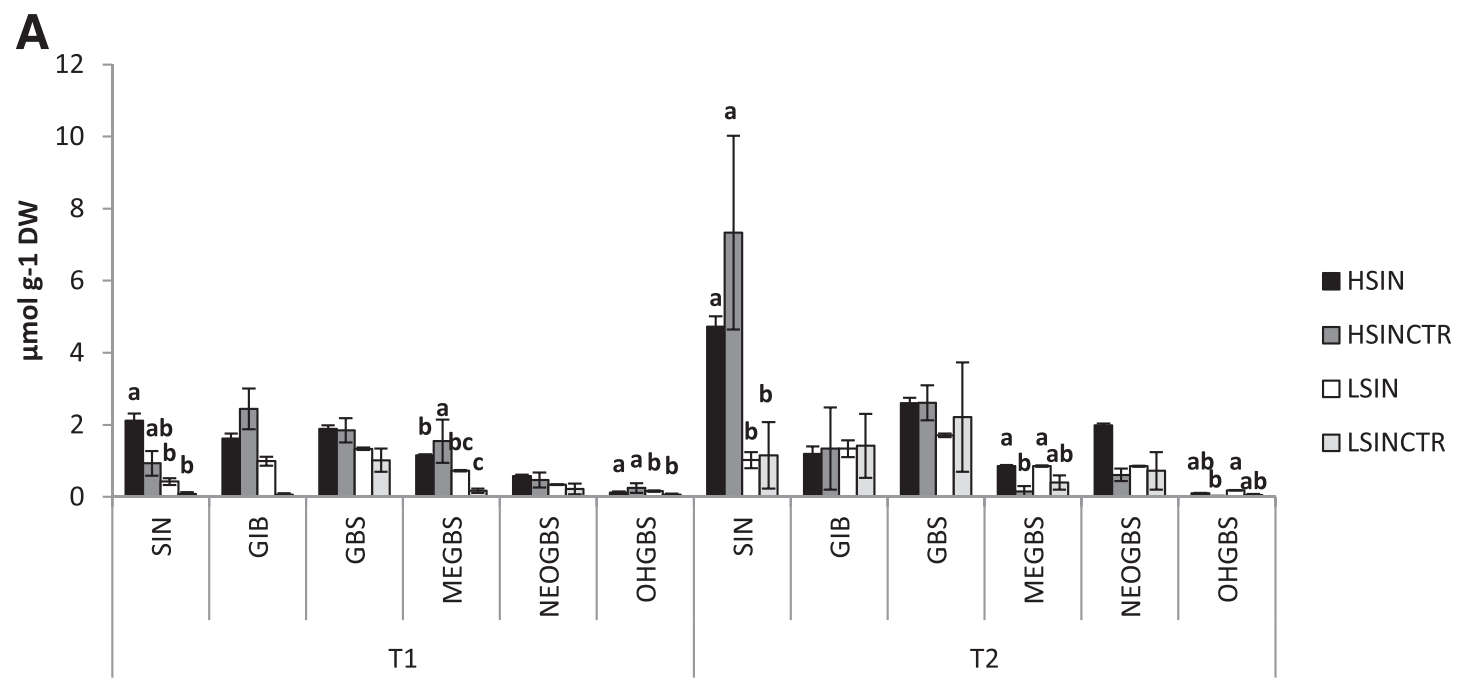

B
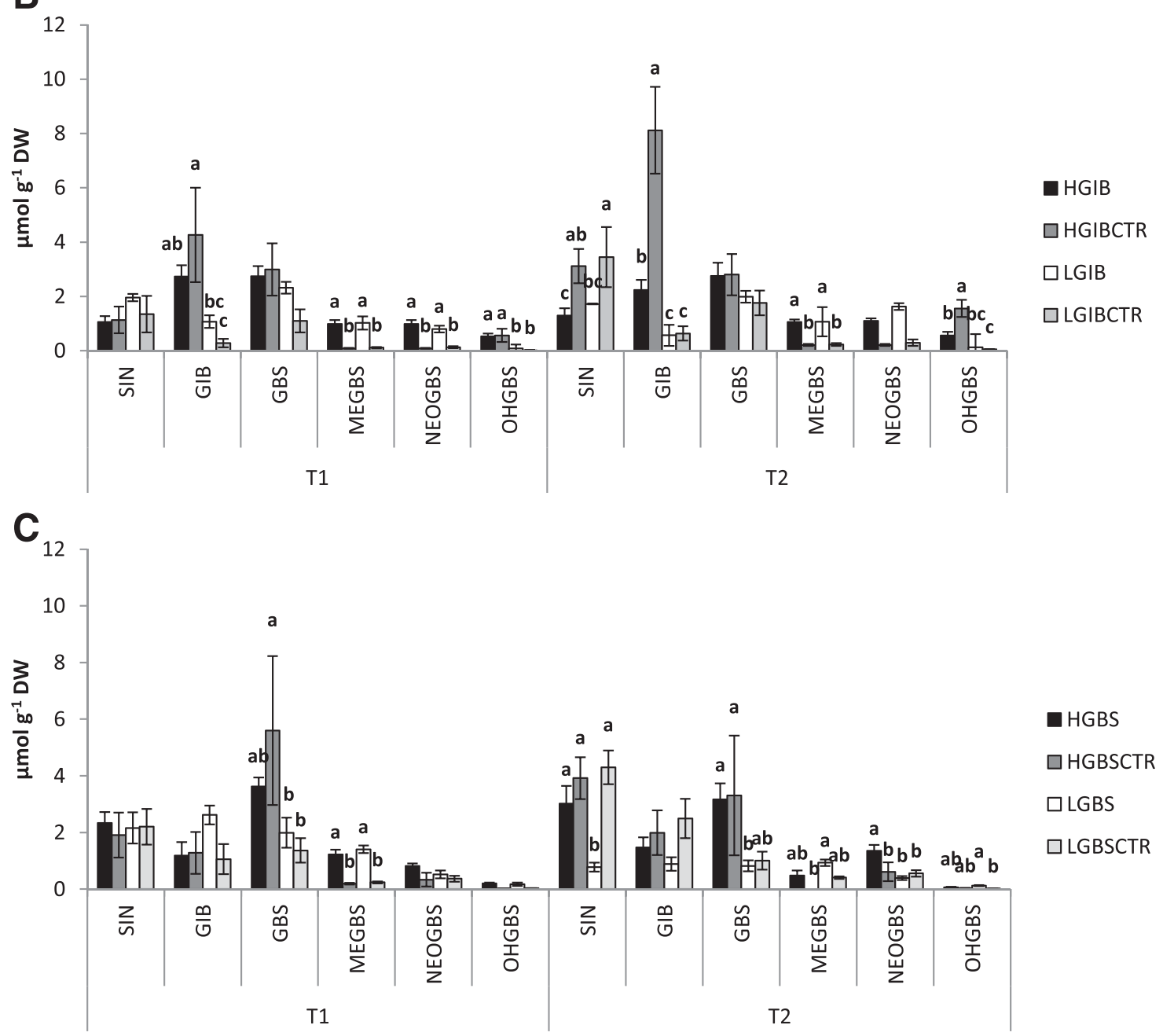

Fig. 1. Comparison of the content of glucosinolates in kale plants inoculated with Sclerotinia sclerotiorum and control plants in two trials (T1 and T2) in A, SINdivergent selection; B, GIB-divergent selection; and $\mathbf{C}$, GBS-divergent selection. Values are the average of 30 replicates \pm standard error. Means with different letters are significantly different at $\alpha \leq 0.05$ according to Fisher's protected least significant difference test. DW = dry weight; SIN = sinigrin, 2-propenyl; GIB = glucoiberin, 3-methylsulphinylpropyl; GBS = 3-indolylmethyl, glucobrassicin; OHGBS = hidroxyglucobrassicin, 4-hydroxy-3-indolylmethyl; NEOGBS = neoglucobrassicin, 1-methoxy-3-indolylmethyl; and MEGBS = methoxyglucobrassicin, 4-methoxy-3-indolylmethyl. 


\section{DISCUSSION}

We demonstrated that genotypes selected for higher GSL content invariably had a greater concentration of the target GSL compared with the corresponding genotypes selected for low GSL content. It is established that changes in a single GSL gene may lead to pleiotropic effects on the concentrations of other GSLs. When divergent selection modifies the content of SIN, effects on the
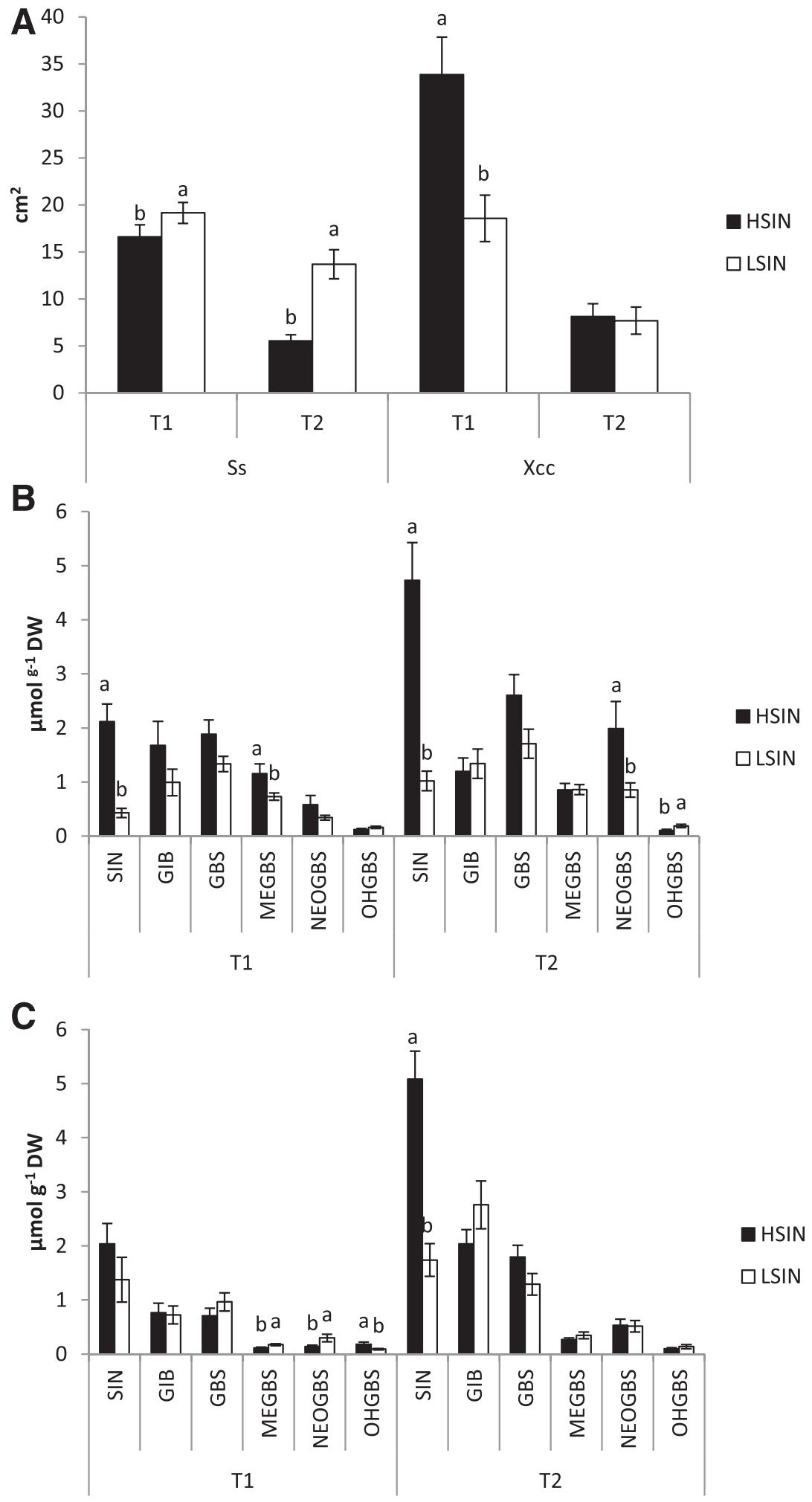

Fig. 2. Effect of high (HSIN) and low (LSIN) SIN content in a selection of kale genotypes on A, lesion area after inoculation with Sclerotinia sclerotiorum (Ss) or Xanthomonas campestris pv. campestris (Xcc) in two trials (T1 and T2); B, content of SIN, GIB, GBS, and minor glucosinolates (GSLs) in the HSIN and LSIN genotypes inoculated with S. sclerotiorum; and C, content of SIN, GIB, GBS, and minor GSLs in HSIN and LSIN genotypes inoculated with X. campestris pv. campestris. Values are the average of 30 replicates \pm standard error. Means with different letters are significantly different at $\alpha \leq 0.05$ according to Fisher's protected least significant difference test. DW = dry weight; SIN = sinigrin, 2-propenyl; GIB = glucoiberin, 3-methylsulphinylpropyl; GBS = 3-indolylmethyl, glucobrassicin; OHGBS = hidroxyglucobrassicin, 4-hydroxy-3-indolylmethyl; NEOGBS = neoglucobrassicin, 1-methoxy-3-indolylmethyl; and MEGBS = methoxyglucobrassicin, 4-methoxy-3-indolylmethyl. 
content of GIB, GBS, or other GSLs may also occur (Sotelo et al. 2016). Therefore, we included minor GSLs present in kale leaves for each selection in studies of the relationship with disease severity. We observed differential performance of the divergent selections after inoculation with $S$. sclerotiorum or $X$. campestris pv. campestris. Genotypes selected for high SIN content were more resistant to $S$. sclerotiorum and genotypes selected for high GBS content were more resistant to $X$. campestris pv. campestris.
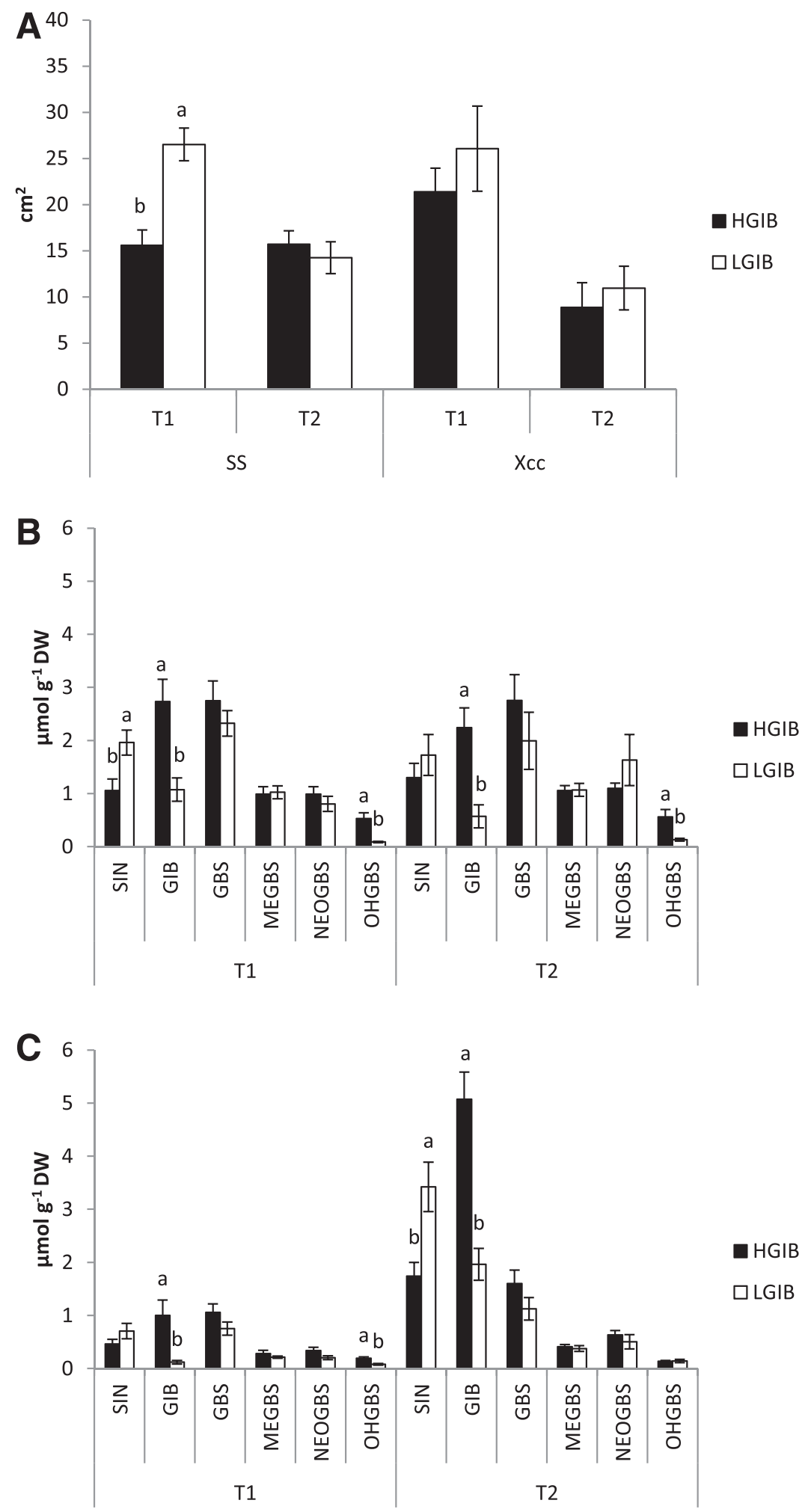

Fig. 3. Effect of high (HGIB) and low (LGIB) GIB content in a selection of kale genotypes on A, lesion area after inoculation with Sclerotinia sclerotiorum (Ss) or Xanthomonas campestris pv. campestris (Xcc) in two trials (T1 and T2); B, content of SIN, GIB, GBS, and minor glucosinolates (GSLs) in the HGIB and LGIB genotypes inoculated with S. sclerotiorum; and C, content of SIN, GIB, GBS, and minor GSLs in HGIB and LGIB genotypes inoculated with X. campestris pv. campestris. Values are the average of 30 replicates \pm standard error. Means with different letters are significantly different at $\alpha \leq 0.05$ according to Fisher's protected least significant difference test. DW = dry weight; SIN = sinigrin, 2-propenyl; GIB = glucoiberin, 3-methylsulphinylpropyl; GBS = 3-indolylmethyl, glucobrassicin; OHGBS = hidroxyglucobrassicin, 4-hydroxy-3-indolylmethyl; NEOGBS = neoglucobrassicin, 1-methoxy-3-indolylmethyl; and MEGBS = methoxyglucobrassicin, 4-methoxy-3-indolylmethyl. 
However, the effect of GIB content was not clear, because the relationship with lesion area was trial dependent in the S. sclerotiorum experiment, and there was no relationship with lesion area when genotypes were inoculated with $X$. campestris pv. campestris.
Our results showed that the aliphatic GSL SIN inhibited the growth of $S$. sclerotiorum (i.e., HSIN genotypes had smaller lesions [less severe disease]). These results are congruent with those of a previous report showing a role of SIN in reducing severity of disease
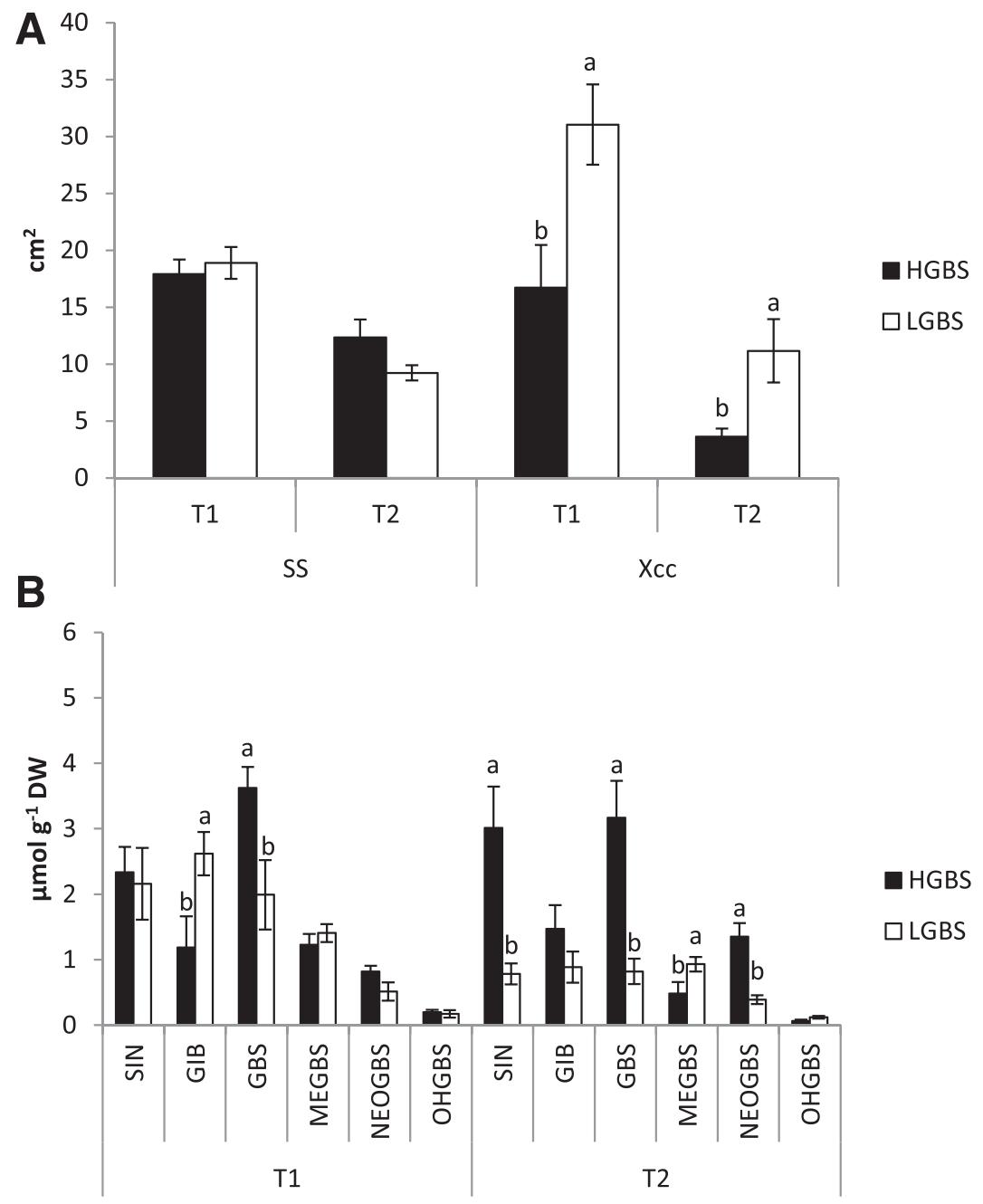

HGBS

$\square$ LGBS

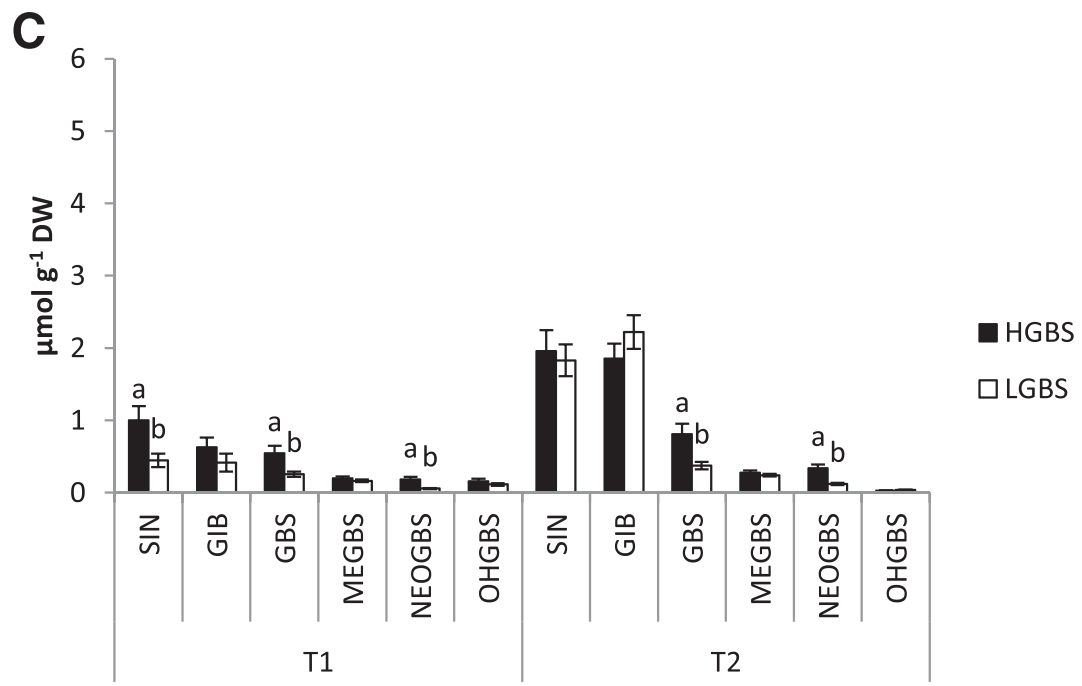

Fig. 4. Effect of high (HGBS) and low (LGBS) GBS content in a selection of kale genotypes on A, lesion area after inoculation with Sclerotinia sclerotiorum (Ss) or Xanthomonas campestris pv. campestris (Xcc) in two trials (T1 and T2); B, content of SIN, GIB, GBS, and minor glucosinolates (GSLs) in the HGBS and LGBS genotypes inoculated with S. sclerotiorum; and C, content of SIN, GIB, GBS, and minor GSLs in HGBS and LGBS genotypes inoculated with X. campestris pv. campestris. Values are the average of 30 replicates \pm standard error. Means with different letters are significantly different at $\alpha \leq 0.05$ according to Fisher's protected least significant difference test. DW = dry weight; SIN = sinigrin, 2-propenyl; GIB = glucoiberin, 3-methylsulphinylpropyl; GBS = 3-indolylmethyl, glucobrassicin; OHGBS = hidroxyglucobrassicin, 4-hydroxy-3-indolylmethyl; NEOGBS = neoglucobrassicin, 1-methoxy-3-indolylmethyl; and MEGBS = methoxyglucobrassicin, 4-methoxy-3-indolylmethyl. 
(Stotz et al. 2011). The authors reported that content of aliphatic GSLs in Arabidopsis were correlated with changes in susceptibility to $S$. sclerotiorum. In addition, when genotypes of the SIN selection were inoculated with $S$. sclerotiorum, the concentrations of two indolic GSLs (NEOGBS and MEGBS) were significantly higher in HSIN than in LSIN. Therefore, the resistance observed with the SIN selection against S. sclerotiorum may be attributed to a combined effect of the aliphatic SIN and the

TABLE 1. Results of an analysis of variance performed to compare the content of glucosinolates in kale plants either inoculated with Sclerotinia sclerotiorum or noninoculated as control plants in two trials (T1 and T2)

\begin{tabular}{|c|c|c|c|c|c|c|c|c|c|c|c|c|}
\hline \multirow[b]{2}{*}{ Selection $^{\mathrm{b}}$} & \multicolumn{6}{|c|}{ T1 glucosinolates } & \multicolumn{6}{|c|}{ T2 glucosinolates } \\
\hline & SIN & GIB & GBS & MEGBS & NEOGBS & OHGBS & SIN & GIB & GBS & MEGBS & NEOGBS & OHGBS \\
\hline \multicolumn{13}{|l|}{ SIN } \\
\hline$F$ value & 13.1 & 2.4 & 2.1 & 6.8 & 1.2 & 3.5 & 12.9 & 0.5 & 1.2 & 3.2 & 2.4 & 3.6 \\
\hline \multicolumn{13}{|l|}{ GIB } \\
\hline$F$ value & 2.6 & 7.7 & 2.1 & 7.00 & 4.8 & 7.6 & 3.7 & 29.4 & 0.7 & 10.7 & 2.5 & 14.4 \\
\hline$P$ value & 0.06 & $<0.001$ & 0.1 & $<0.001$ & 0.005 & $<0.001$ & 0.02 & $<0.001$ & 0.6 & $<0.001$ & 0.07 & $<0.001$ \\
\hline
\end{tabular}

a SIN = sinigrin, 2-propenyl; GIB = glucoiberin, 3-methylsulphinylpropyl; GBS = 3-indolylmethyl, glucobrassicin; OHGBS = hidroxyglucobrassicin, 4-hydroxy3-indolylmethyl; NEOGBS = neoglucobrassicin, 1-methoxy-3-indolylmethyl; and MEGBS = methoxyglucobrassicin, 4-methoxy-3-indolylmethyl. Results presented are for each of the three divergent selections for different glucosinolate types (SIN, GIB, and GBS; content of MEGBS, NEOGBS, and OHGBS were also measured in the three divergent selections).

b $F$ value of the $F$ distribution and $P$ value indicating the probability of the effect.

TABLE 2. Results of an analysis of variance performed to compare the content of glucosinolates and lesion area (Area) in kale plants either inoculated with Sclerotinia sclerotiorum (S. scler) or Xanthomonas campestris pv. campestris (X. camp) or noninoculated as control plants in two trials (T1 and T2) ${ }^{\mathrm{a}}$

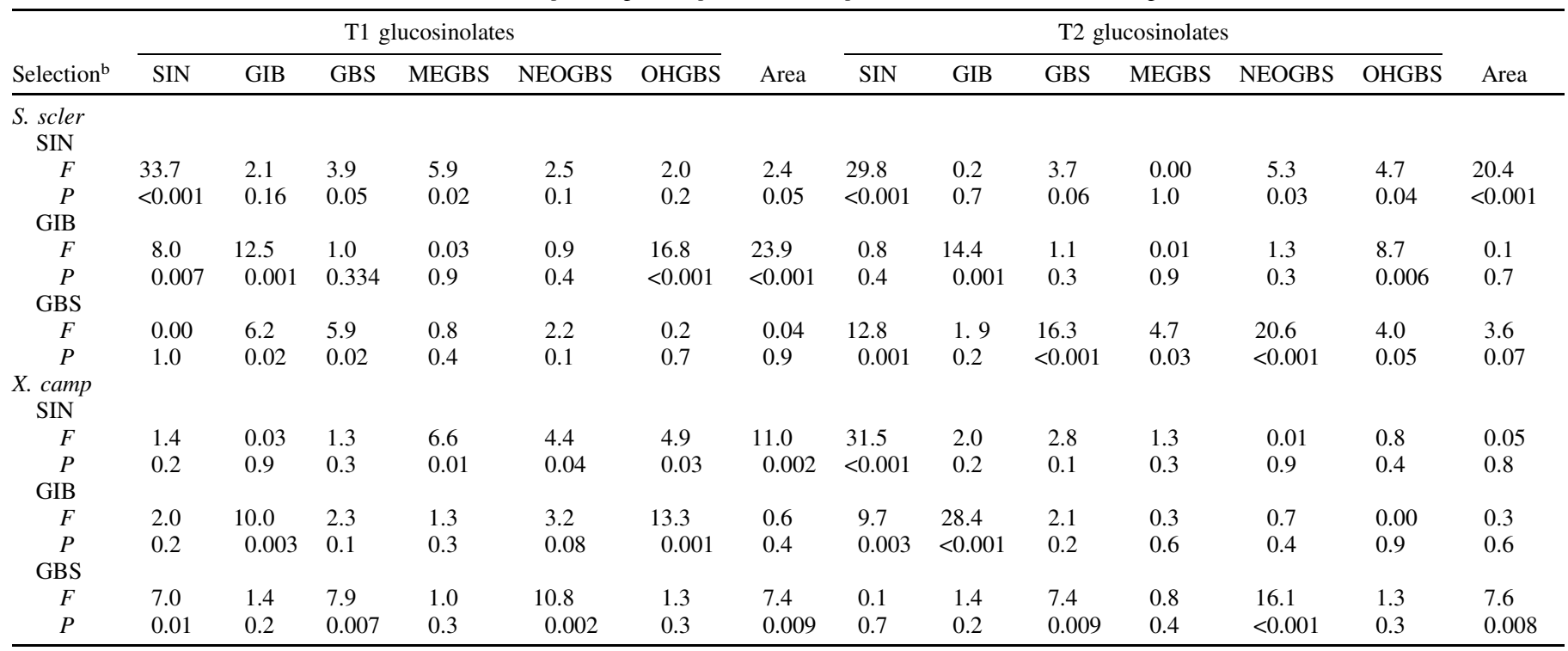

a SIN = sinigrin, 2-propenyl; GIB = glucoiberin, 3-methylsulphinylpropyl; GBS = 3-indolylmethyl, glucobrassicin; OHGBS = hidroxyglucobrassicin, 4-hydroxy3-indolylmethyl; NEOGBS = neoglucobrassicin, 1-methoxy-3-indolylmethyl; and MEGBS = methoxyglucobrassicin, 4-methoxy-3-indolylmethyl. Results presented are for each of the three divergent selections for different glucosinolate types (SIN, GIB, and GBS; content of MEGBS, NEOGBS, and OHGBS were also measured in the three divergent selections).

b $F$ value of the $F$ distribution and $P$ value indicating the probability of the effect.

TABLE 3. Results of an analysis of variance performed to compare the content of glucosinolates and lesion area (Area) in kale plants either inoculated with Sclerotinia sclerotiorum (S. scler) or Xanthomonas campestris pv. campestris (X. camp) or noninoculated as control plants in two trials (T1 and T2) ${ }^{\mathrm{a}}$

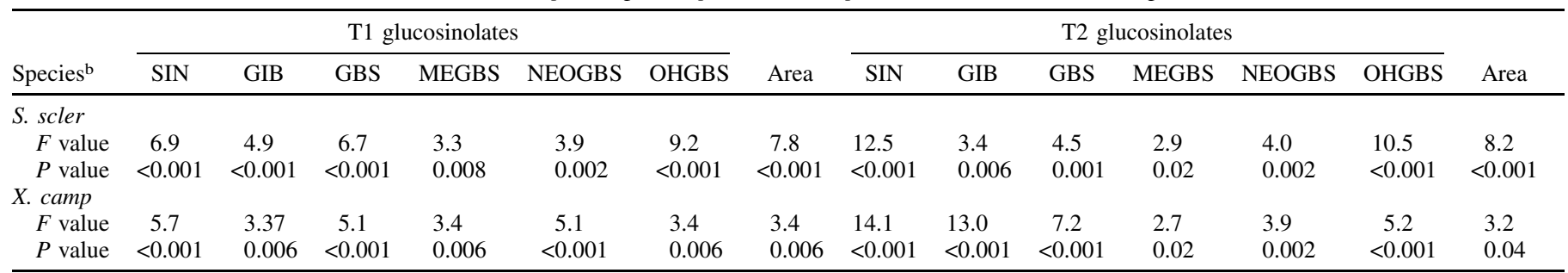

a SIN = sinigrin, 2-propenyl; GIB = glucoiberin, 3-methylsulphinylpropyl; GBS = 3-indolylmethyl, glucobrassicin; OHGBS = hidroxyglucobrassicin, 4-hydroxy3-indolylmethyl; NEOGBS = neoglucobrassicin, 1-methoxy-3-indolylmethyl; and MEGBS = methoxyglucobrassicin, 4-methoxy-3-indolylmethyl. Results presented are combined through the three divergent selections for different glucosinolate types (SIN, GIB, and GBS; content of MEGBS, NEOGBS, and OHGBS were also measured in the three divergent selections).

${ }^{\mathrm{b}} F$ value of the $F$ distribution and $P$ value indicating the probability of the effect. 
indolic GSLs that, together, enhanced the plant defense response.

The effect of GIB selection was not clear, because the response of the HGIB and LGIB genotypes was dependent upon the trial when plants were inoculated with $S$. sclerotiorum. Previous reports indicate that different alleles at the GSL-ALK locus are selected for in the divergent selections for SIN and GIB (Sotelo et al. 2016). GSL-ALK alkenized GIB to render SIN. Therefore, when the content of one increases, the content of the other decreases. SIN inhibited the growth of $S$. sclerotiorum, as demonstrated in

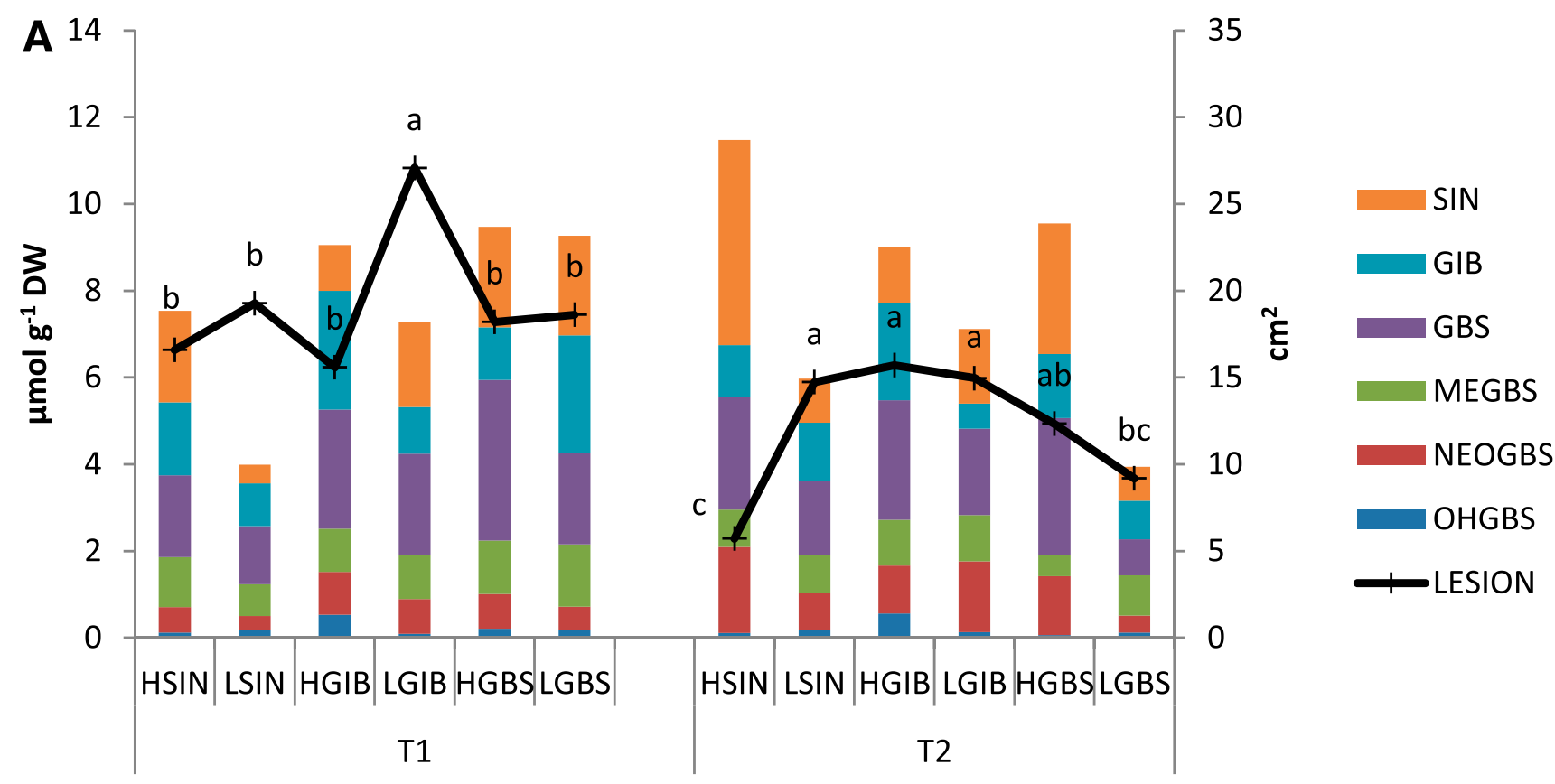

B

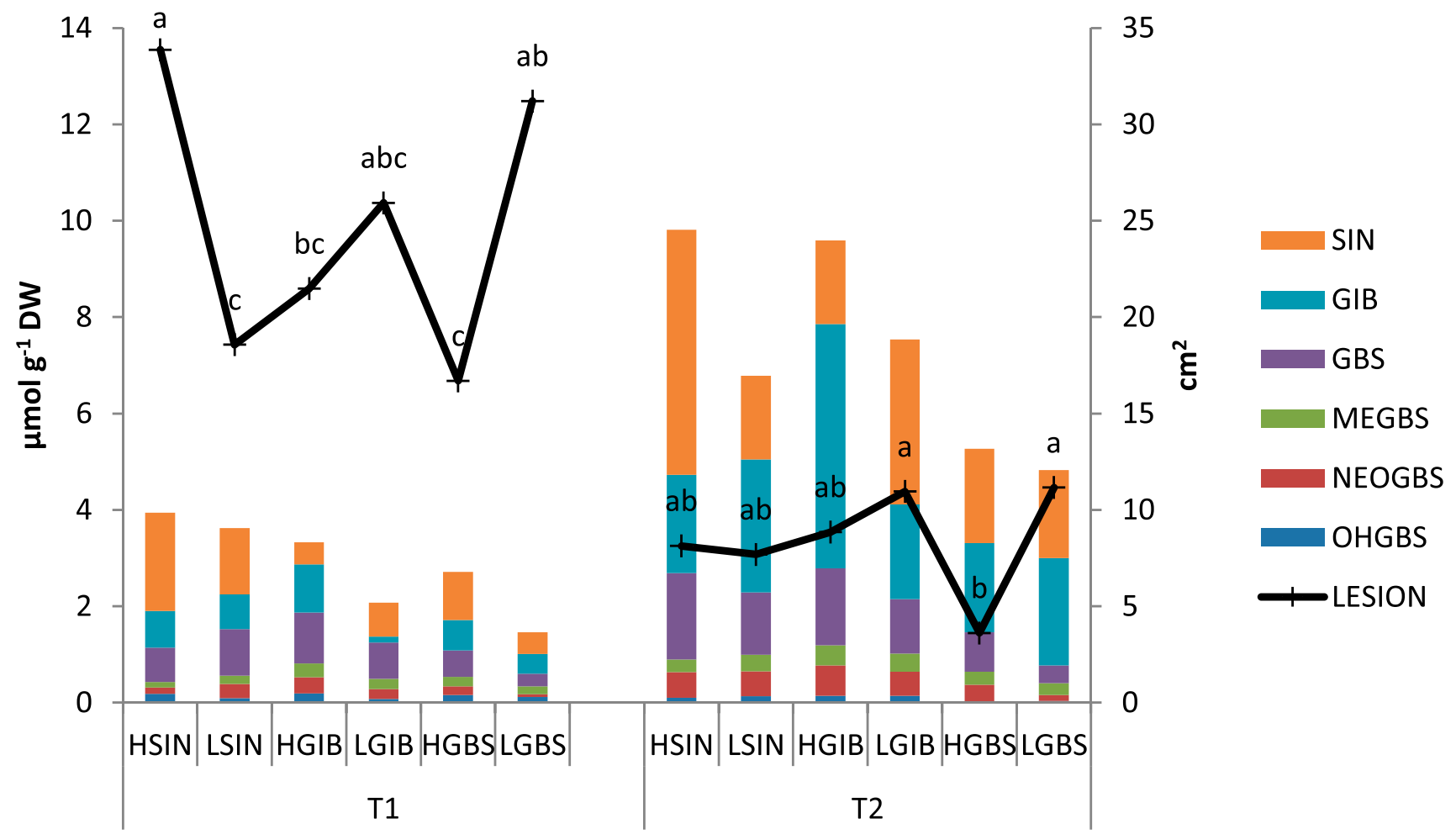

Fig. 5. Glucosinolate content of three divergent selections of kale, each of which were selected for high (HSIN, HGIB, and HGBS) or low (LSIN, LGIB, and LGBS) glucosinolate content, and the lesion area on each selection after inoculation with $\mathbf{A}$, Sclerotinia sclerotiorum or $\mathbf{B}$, Xanthomonas campestris pv. campestris in two trials (T1 and T2). Values are the average of 30 replicates \pm standard error. Lesion means with different letters are significantly different at $\alpha \leq 0.05$ according to Fisher's protected least significant difference test. DW = dry weight; SIN = sinigrin, 2-propenyl; GIB = glucoiberin, 3-methylsulphinylpropyl; GBS = 3-indolylmethyl, glucobrassicin; OHGBS = hidroxyglucobrassicin, 4-hydroxy-3-indolylmethyl; NEOGBS = neoglucobrassicin, 1-methoxy-3-indolylmethyl; and MEGBS = methoxyglucobrassicin, 4-methoxy-3-indolylmethyl. 
SIN selection. HGIB had a lower concentration of SIN than LGIB. This could explain why the selection to modify GIB content did not have a clear effect on lesion area as a result of infection by S. sclerotiorum.

Several reports indicate that indolic GSLs are inhibitory against S. sclerotiorum in vivo (Li et al. 1999; Zhang et al. 2015). However, we did not find a clear effect of the HGBS genotypes reducing lesion area caused by $S$. sclerotiorum. Lack of an effect may be due to variations in the GBS content in the divergent selection used in our study. Such variations are reported to occur in expression of the CYP81F2 gene (Sotelo et al. 2016). The CYP81F2 gene converts GBS into OHGBS, and should not affect other metabolic pathways. However, research that relates indolic GSLs to resistance to $S$. sclerotiorum in Arabidopsis through mutations in the UGT74B1 or CYP79B genes indicates that they affect not only the content of indolic GSLs but also other plant immune system metabolites (Zhang et al. 2015). Therefore, it is not clear whether the effect can be attributed to an increase in total indolic GSLs or to the induction of other plant immune system metabolites (i.e., camalexin or auxins). Thus, defense responses against $S$. sclerotiorum are probably regulated by a complex network of hormonal and signaling pathways (i.e., jasmonic acid, ethylene, and abscisic acid) promoting the production of GSLs and other metabolites, working together to enhance plant resistance.

Little is known about the mechanisms of resistance to $X$. campestris pv. campestris in Brassica crops. Aires et al. (2011) concluded that both aliphatic and indolic GSLs may play a complex role in the defense, depending on the species of Brassica. Velasco et al. (2013) reported that the aliphatic gluconapin (3-butenyl) can protect $B$. rapa against $X$. campestris pv. campestris. We found that HGBS plants were more resistant to $X$. campestris pv. campestris, whereas the aliphatics SIN and GIB did not have an effect in reducing lesion area. Overall, these findings suggest that an increment of individual GSLs does not always lead to enhanced disease resistance.

GSLs are present in all tissues of the plants. They are produced constitutively but are also inducible in response to biotic stress (Brader et al. 2001; Buxdorf et al. 2013). To explore the relationship between the endogenous concentration of GSLs and the induced component in GSLs as a result of infection by S. sclerotiorum, the content of GSLs in the high and low selections of each genotypes were compared with that of the controls. Aires et al. (2011) related the suppression of de novo synthesis of defense compounds and the inhibition of myrosinase activity with the mode of infection by a necrotrophic pathogen. Whereas hypersensitive cell death can be an effective defense strategy against biotrophs, it may not be efficient against necrotrophic pathogens. However, a reduction in lesion size caused by $S$. sclerotiorum in oilseed rape (B. napus) was related to induction of indolic and aromatic GSLs (Li et al. 1999). Induced synthesis of GSLs may be related to the plant genotype and to its interaction with specific pathogens. Similar to the observations of Li et al. (1999), we found that indolic GSLs were induced after inoculation with $S$. sclerotiorum in all three selections but induction was most pronounced with the GIB genotype. However, aliphatic GSLs were repressed in the GIB genotype. These apparently opposing responses among GSLs could contribute to the poor performance of GIB against S. sclerotiorum.

The process of GSL hydrolysis may be related to the differential performance of the divergent selections after infection with $S$. sclerotiorum and X. campestris pv. campestris. Myrosinases and GSLs are localized in two different specialized cells. When there is tissue destruction (for example if a necrotroph infects), the GSLs are exposed to the myrosinases which hydrolyze the $\beta$-Dthioglucosidic bond of the GSL to release $\beta$-D-glucose and an unstable aglucone (Calmes et al. 2015; Shirakawa and HaraNishimura 2018). The aglucone spontaneously forms an ITC by rearrangement or decomposes to a nitrile. Formation of ITCs is favored at $\mathrm{pH}$ values of 5 to 6 , whereas nitriles are preferentially formed at a $\mathrm{pH}<4$ or at increased $\mathrm{Fe}^{2+}$ concentrations (Hanschen et al. 2018). In addition, epithiospecific proteins may promote the formation of nitriles and epithionitriles by modulating myrosinase activity. As a consequence, tissue disruption typically leads to the release of complex mixtures of GSL breakdown products (Burow et al. 2009; Hanschen et al. 2018). We measured the quantity of intact GSLs and not their degradation products after inoculation. We would expect a reduction in the concentration of GSLs when comparing inoculated plants with controls as a part of the process of degradation. However, this does not always happen, because several GSLs were induced upon S. sclerotiorum infection. Therefore, the concentrations of GSLs in inoculated plants are the product of their degradation and of their induced synthesis upon infection.

When comparing the three divergent selections, the HSIN and HGBS plants had greater resistance to $S$. sclerotiorum and $X$. campestris pv. campestris, respectively. However, the resistance is not only related to the content of GSLs: in the SIN-divergent selection group, the content of SIN was related to resistance. However, the relationship with resistance was not observed in the GBS selection, where the HGBS plants had a higher content of SIN compared with the LGBS plants. Other processes in the plant could modify the plant response because the three divergent selections were performed independently, resulting in different genetic backgrounds for each GSL selection. Modifying the content of GSLs could affect other processes in the plant.

Conclusions. The toxic effect of GSL-derived products has been demonstrated with a broad range of fungi and bacteria by in vitro experiments. However, the role of GSLs in plant defense is more complex. Increasing the content of individual GSLs does not always result in reduced disease severity. The effect of GSL is clearly dependent on the pathogen and the chemical class of the GSL. We observed that the aliphatic SIN was inhibitory to disease caused by $S$. sclerotiorum but the indolic GBS was inhibitory to disease caused by X. campestris pv. campestris. Additional factors, including the proportions of other metabolites in the host during the pathogen infection, could modulate the response of the plant. Differential performance of genotypes across divergent selections cannot be attributed solely to variation in GSL content. We conclude that other processes in the plant that affect disease response were probably modified when the divergent selections were developed.

\section{LITERATURE CITED}

Aires, A., Dias, C. S. P., Carvalho, R., Oliveira, M. H., Monteiro, A. A., Simoes, M. V., Rosa, E. A. S., Bennett, R. N., and Saavedra, M. J. 2011. Correlations between disease severity, glucosinolate profiles and total phenolics and Xanthomonas campestris pv. campestris inoculation of different Brassicaceae. Sci. Hortic. (Amsterdam) 129:503-510.

Aires, A., Mota, V. R., Saavedra, M. J., Monteiro, A. A., Simoes, M., Rosa, E. A. S., and Bennett, R. N. 2009. Initial in vitro evaluations of the antibacterial activities of glucosinolate enzymatic hydrolysis products against plant pathogenic bacteria. J. Appl. Microbiol. 106:2096-2105.

Brader, G., Mikkelsen, M. D., Halkier, B. A., and Palva, E. T. 2006. Altering glucosinolate profiles modulates disease resistance in plants. Plant J. 46: 758-767.

Brader, G., Tas, E., and Palva, E. T. 2001. Jasmonate-dependent induction of indole glucosinolates in Arabidopsis by culture filtrates of the nonspecific pathogen Erwinia carotovora. Plant Physiol. 126:849-860.

Burow, M., Losansky, A., Müller, R., Plock, A., Kliebenstein, D. J., and Wittstock, U. 2009. The genetic basis of constitutive and herbivore-induced ESP-independent nitrile formation in Arabidopsis. Plant Physiol. 149:561-574.

Buxdorf, K., Yaffe, H., Barda, O., and Levy, M. 2013. The effects of glucosinolates and their breakdown products on necrotrophic fungi. PLoS One 8: e70771.

Calmes, B., N'Guyen, G., Dumur, J., Brisach, C. A., Campion, C., Iacomi, B., Pigne, S., Dias, E., Macherel, D., Guillemette, T., and Simoneau, P. 2015. Glucosinolate-derived isothiocyanates impact mitochondrial function in fungal cells and elicit an oxidative stress response necessary for growth recovery. Front. Plant Sci. 6:414.

Dufour, V., Stahl, M., and Baysse, C. 2015. The antibacterial properties of isothiocyanates. Microbiology-Sgm 161:229-243.

Fox, J. and Bouchet-Valata, M. 2018. Rcmdr: R Commander. R package version 2.5-1. https://cran.r-project.org/web/packages/Rcmdr/index.html 
Frerigmann, H., Pislewska-Bednarek, M., Sanchez-Vallet, A., Molina, A., Glawischnig, E., Gigolashvili, T., and Bednarek, P. 2016. Regulation of pathogen-triggered tryptophan metabolism in Arabidopsis thaliana by MYB transcription factors and indole glucosinolate conversion products. Mol. Plant 9:682-695.

Giamoustaris, A., and Mithen, R. 1997. Glucosinolates and disease resistance in oilseed rape (Brassica napus ssp oleifera). Plant Pathol. 46:271-275.

Hanschen, F. S., Pfitzmann, M., Witzel, K., Stuetzel, H., Schreiner, M., and Zrenner, R. 2018. Differences in the enzymatic hydrolysis of glucosinolates increase the defense metabolite diversity in 19 Arabidopsis thaliana accessions. Plant Physiol. Biochem. 124:126-135.

Johansson, O. N., Fantozzi, E., Fahlberg, P., Nilsson, A. K., Buhot, N., Tor, M., and Andersson, M. X. 2014. Role of the penetration-resistance genes PEN1, PEN2 and PEN3 in the hypersensitive response and race-specific resistance in Arabidopsis thaliana. Plant J. 79:466-476.

Kayum, M. A., Kim, H.-T., Nath, U. K., Park, J.-I., Kho, K. H., Cho, Y.-G., and Nou, I.-S. 2016. Research on biotic and abiotic stress related genes exploration and prediction in Brassica rapa and B. oleracea-A review. Plant Breed. Biotechnol. 4:135-144.

Kliebenstein, D. J., Lambrix, V. M., Reichelt, M., Gershenzon, J., and Mitchell-Olds, T. 2001. Gene duplication in the diversification of secondary metabolism: Tandem 2-oxoglutarate-dependent dioxygenases control glucosinolate biosynthesis in Arabidopsis. Plant Cell 13:681-693.

Li, D. G., Shu, Y. A., Li, P. L., Zhang, W. B., Ni, H. W., and Cao, Y. S. 2013. Synthesis and structure-activity relationships of aliphatic isothiocyanate analogs as antibiotic agents. Med. Chem. Res. 22:3119-3125.

Li, Y., Kiddle, G., Bennett, R. N., and Wallsgrove, R. M. 1999. Local and systemic changes in glucosinolates in Chinese and European cultivars of oilseed rape (Brassica napus L.) after inoculation with Sclerotinia sclerotiorum (stem rot). Ann. Appl. Biol. 134:45-58.

Padilla, G., Cartea, M. E., and Ordás, A. 2007. Comparison of several clustering methods in grouping kale landraces. J. Am. Soc. Hortic. Sci. 132:387-395.

Pastorczyk, M., and Bednarek, P. 2016. The function of glucosinolates and related metabolites in plant innate immunity. Pages 171-198 in: Advances in Botanical Research, Vol. 80, Glucosinolates. S. Kopriva, ed. Elsevier, Ltd, London, U.K.

R Core Team. 2018. R: A Language and Environment for Statistical Computing. R Foundation for Statistical Computing, Vienna, Austria. http:// wWw.R-project.org/
Shirakawa, M., and Hara-Nishimura, I. 2018. Specialized vacuoles of myrosin cells: Chemical defense strategy in Brassicales plants. Plant Cell Physiol. 59:1309-1316.

Sotelo, T., Lema, M., Soengas, P., Cartea, M. E., and Velasco, P. 2014a. In vitro activity of glucosinolates and their degradation products against Brassica-pathogenic bacteria and fungi. Appl. Environ. Microbiol. 81: 432-440.

Sotelo, T., Soengas, P., Velasco, P., Rodriguez, V. M., and Cartea, M. E. 2014b. Identification of metabolic QTLs and candidate genes for glucosinolate synthesis in Brassica oleracea leaves, seeds and flower buds. PLoS One 9: e91428.

Sotelo, T., Velasco, P., Soengas, P., Rodriguez, V. M., and Cartea, M. E. 2016. Modification of leaf glucosinolate contents in Brassica oleracea by divergent selection and effect on expression of genes controlling glucosinolate pathway. Front. Plant Sci. 7:1012. https://www.frontiersin.org/articles/ 10.3389/fpls.2016.01012/full

Stahl, E., Bellwon, P., Huber, S., Schlaeppi, K., Bernsdorff, F., Vallat-Michel, A., Mauch, F., and Zeier, J. 2016. Regulatory and functional aspects of indolic metabolism in plant systemic acquired resistance. Mol. Plant 9: 662-681.

Stotz, H. U., Sawada, Y., Shimada, Y., Hirai, M. Y., Sasaki, E., Krischke, M., Brown, P. D., Saito, K., and Kamiya, Y. 2011. Role of camalexin, indole glucosinolates, and side chain modification of glucosinolate-derived isothiocyanates in defense of Arabidopsis against Sclerotinia sclerotiorum. Plant J. 67:81-93.

Tortosa, M., Velasco, P., Alfonso, D., Padilla, G., Ríos, D., and Soengas, P. 2017. Characterization of a Spanish Brassica oleracea collection by using molecular and biochemical markers. Sci. Hortic. (Amsterdam) 219: 344-350.

Velasco, P., Lema, M., Francisco, M., Soengas, P., and Elena Cartea, M. 2013. In vivo and in vitro effects of secondary metabolites against Xanthomonas campestris pv. campestris. Molecules 18:11131-11143.

Zhang, Y., Huai, D., Yang, Q., Cheng, Y., Ma, M., Kliebenstein, D. J., and Zhou, Y. 2015. Overexpression of three glucosinolate biosynthesis genes in Brassica napus identifies enhanced resistance to Sclerotinia sclerotiorum and Botrytis cinerea. PLoS One 10:e0140491.

Zukalova, H., and Vasak, J. 2002. The role and effects of glucosinolates of Brassica species-A review. Rostl. Vyroba 48:175-180. 